\title{
Perspectives on the Early Quality of Evidence Guiding the Therapeutic Management of SARS-CoV-2: A Systematic Literature Review
}

\author{
Kaushik Subramanian - Anuradha Nalli • Vinitha Senthil • \\ Saurabh Jain · Aravind Nayak · Amit Bhat
}

Received: June 30, 2020 / Published online: August 18, 2020

(C) Springer Healthcare Ltd., part of Springer Nature 2020

\begin{abstract}
Background: The severe acute respiratory syndrome coronavirus 2 (SARS-CoV-2) outbreak is a serious health concern. Repurposing of existing drugs indicated for other conditions seems to be the first choice for immediate therapeutic management. The quality of early evidence favoring the different treatment options needs to be apprised for informed decision-making.

Methods: In this systematic literature review, we apprised the quality of available evidence for different therapeutic options and also the basis for different treatment guidelines. To include all studies that are in different stages of publication, we also included studies from the preprint servers BioRxiv and MedRxiv and published studies from PubMed.
\end{abstract}

Digital Features To view digital features for this article go to https://doi.org/10.6084/m9.figshare.12722258.

K. Subramanian · A. Nalli · V. Senthil · S. Jain

A. Nayak

Indegene Pvt Ltd, Bengaluru, India

A. Bhat $(\bowtie)$

Indegene Lifesystems Consulting (Shanghai) Co.

Ltd, Shanghai, China

e-mail: amit.bhat@indegene.com
Results: We retrieved 5621 articles and included 22 studies for the systematic review. Based on our study, chloroquine/hydroxychloroquine, either alone or in combination with azithromycin, remdesivir, corticosteroids, convalescent sera, ritonavir/lopinavir, tocilizumab and arbidol were evaluated as therapeutic options. The data from different study designs reveal contradictory findings except for convalescent sera for which the evidence available is only from case series. Based on this early evidence, various national guidelines recommend remdesivir, convalescent sera, corticosteroids and hydroxychloroquine in different subsets of patients.

Conclusion: Establishing consensus with respect to the end points to be assessed for respiratory viruses may enhance the quality of evidence in case of future pandemics. The systematic review highlighted the lacuna and methodologic deficiency in early clinical evidence and included an update on different therapeutic management guidelines. Further clinical evidence from the ongoing trials may lead to evolution of treatment guidelines with the addition of more therapeutic options.

Keywords: Clinical cure; Evidence; Infectious disease; SARS-CoV-2; Treatment options; Virological cure 


\section{Key Summary Points}

The quality of early evidence favoring the different treatment options needs to be apprised for informed decision-making.

We performed a comprehensive systematic literature search and appraisal of early clinical evidence for the therapeutic management of SARS-CoV-2 infection.

An overview on the difference in recommendations and the evidence base for arriving at the recommendations by various guidelines have been provided.

The insights from quality of early evidence will also assist in mounting a better response to future pandemics.

\section{INTRODUCTION}

The severe acute respiratory syndrome coronavirus 2 (SARS-CoV-2) outbreak, which started as a cluster of pneumonia cases in Wuhan, China, in December 2019 was declared a pandemic by the World Health Organization (WHO) on March 11, 2020 [1]. As per the data from the WHO, as of May 31, 2020, it had infected 5.9 million people globally, with approximately $3,67,000$ people succumbing to the infection [2]. After isolation and sequence analysis, the causative virus was grouped into the coronavirus (CoV) family, consisting of RNA viruses that had already caused three different outbreaks of pneumonia in the last 2 decades. The severe acute respiratory syndrome (SARS) that broke out in 2003 was caused by SARS-CoV, whereas the Middle East respiratory syndrome (MERS) that broke out in 2012 was caused by the MERS-CoV [3]. The viral etiologic agent was named SARS-CoV-2 by the international viral classification commission, and the disease was officially named COVID-19 by the WHO [4].

Clinical observation with SARS-CoV-2 revealed mild illness in a majority of the

patients and severe lung injury or multiorgan failure in approximately $5 \%$ of the patients, with a case fatality ratio of $1.4 \%$ [5]. The pathologic findings in severely or critically ill patients revealed manifestations of shock and sepsis, which is hypothesized to be caused by the virus-induced "cytokine storm" [6]. The levels of proinflammatory cytokines and chemokines, including tumor necrosis factor (TNF)- $\alpha$, interleukin (IL)-1 $\beta$, IL-6, granulocytecolony-stimulating factor, interferon-gammainduced protein-10, monocyte chemoattractant protein-1 and macrophage inflammatory proteins $1-\alpha$, were reported to be elevated from early clinical observations, substantiating the "cytokine storm" hypothesis. In cases with mild infection, the resident macrophages in the lung initiate an inflammatory response culminating in the successful containment of the replication of SARS-CoV-2. However, in patients with severe COVID-19 infection, the pathologic findings revealed the impairment of the epithelial-endothelial barrier, leading to a large exudate into the alveolar cavity [6]. The disruption of the endothelial lining initiates a vicious cycle of tissue damage because of the inflammatory response facilitated by the accumulation of regional macrophages, neutrophils and lymphocytes, resulting in a "cytokine storm." Apart from the epithelial cells, virus multiplication in lung capillary endothelial cells has also been reported, which may lead to exudation of plasma in the alveolar cavity leading to microvascular dysfunction. Abnormal coagulation leading to disseminated intravascular coagulation has also been reported in the majority of fatal cases suggesting coagulopathy due to viral sepsis as a possible terminal clinical manifestation. The suggested reasons for coagulopathy in COVID-19 patients include but are not limited to viral sepsis, cytokine storm and multi-organ failure [4, 7]. The incidence of venous thromboembolism is also reported to be high in COVID-19 patients treated in the intensive care unit (ICU), and hence multiple treatment guidelines recommend prophylactic treatment for venous thromboembolism to minimize fatal outcomes [8].

Elderly patients and patients with comorbidities are more susceptible to severe COVID- 
19 infection [9]. The current case fatality rate of COVID-19 is lower than during the 1918 influenza pandemic but higher than during the 1957 influenza pandemic [10]. However, among the recent outbreaks of $\mathrm{CoV}$ infections (SARS-CoV and MERV), SARS-CoV-2 was found to be much more adoptable to different geographic locations with a higher propensity for person-toperson transfer [11].

The typical response to a pandemic involves both short- and long-term plans to minimize the case fatality rate and reduce the response time for future pandemics [11]. The current strategies for reducing fatality mainly involve symptomatic management and therapeutic interventions [4]. The choices available for therapeutic management were mainly based on immune modulators acting on inflammatory tissue damage and antiviral drugs. Although immune modulators can minimize the inflammatory effects, they may suppress the innate immune responses, leading to delay in viral clearance [11]. Antiviral drugs that were proven to be effective in the treatment of RNA virus infection are currently being explored as possible treatment options. However, in the era of evidence-based medicine, the response to a pandemic typically involves repurposing existing drugs targeting specific steps in the pathogenesis. The early evidence base for the successful treatment of COVID-19 may provide insights into mounting a response to future pandemics. Furthermore, the gaps and drawbacks in the early evidence may also provide insights into mounting appropriate responses with respect to the generation of clinical evidence in case of future pandemics. Unlike previous pandemics, the current one is in the era of evidence-based medicine wherein the responses by various national and international nodal organizations will be based only on the quality of evidence. Furthermore, unlike other therapy areas, in case of pandemics, the treatment guidelines may undergo rapid paradigm shifts as the clinical evidence landscape gains maturity with respect to treatment options and end points for assessing efficacy. Hence, in this present study, we evaluated the quality of early clinical evidence currently guiding the treatment strategies for COVID-19 and the therapeutic recommendations of different treatment guidelines.
We also provided a perspective on the quality of early evidence and its probable utility for future responses to pandemics.

\section{METHODS}

This systematic literature review aimed to answer the following questions: What are the different pharmacologic interventions used in the therapeutic management of patients with COVID-19 infection? What is the quality of the evidence on which the different pharmacologic interventions currently practiced in clinical settings for the therapeutic management of COVID-19 are based? Which drug/pharmacologic interventions used for the therapeutic management of patients with COVID-19 have sufficient evidence to support their use in clinical practice?

\section{Data Sources and Searches}

To ensure the retrieval of all relevant studies, we searched PubMed with a broad key word "COVID-19." We also included COVID-19 studies in preprint servers, such as MedRxiv and BioRxiv (https://connect.biorxiv.org/relate/ content/181). Duplicate studies were removed, and a consolidated Excel sheet was prepared for screening. Any study evaluating a therapeutic drug in in vivo or in vitro model was considered a relevant evidence base and graded based on the hierarchy of an evidence-based pyramid into preclinical, case report, case series, cross-sectional, retrospective cohort, prospective cohort, randomized controlled trials (RCTs), meta-analysis and systematic reviews. This systematic literature review was registered in Prospero (CRD42020180148). This article is based on previously conducted studies and does not contain any studies with human participants or animals performed by any of the authors.

\section{Study Selection}

The systematic review yielded a total of 4027 articles from PubMed and 1591 articles from preprint servers (Fig. 1). Primary screening with 


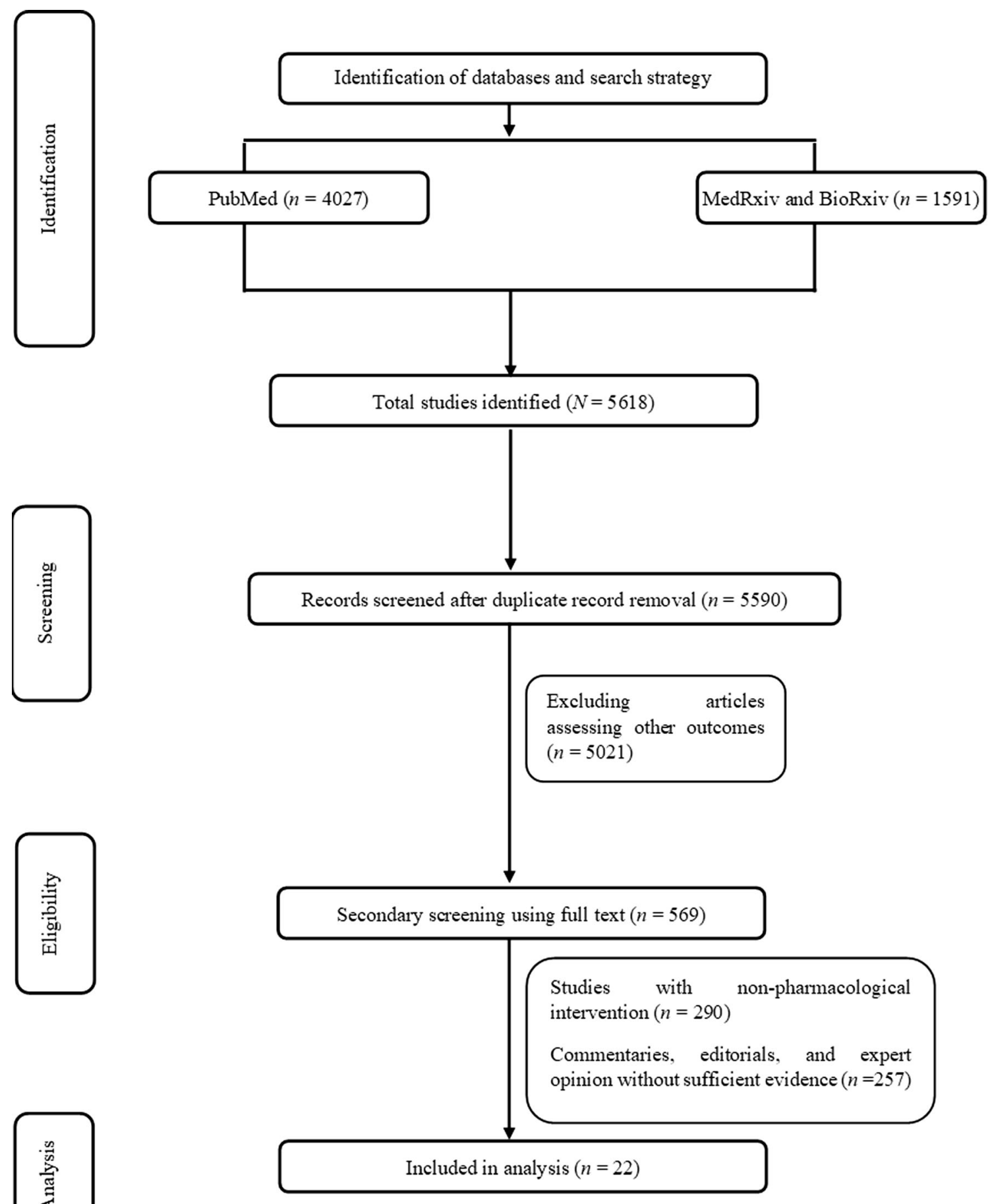

Fig. 1 PRISMA study selection flowchart

the title and abstract revealed a total of 569 relevant articles. On the basis of the full-text screening, a total of 22 studies (Table 1) were included for the evidence synthesis. The studies included for appraisal ranged from case reports to RCTs. 


\section{Data Extraction and Quality Assessment}

The evidence was categorized as very low, low, moderate and strong as per the Grading of Recommendations Assessment, Development and Evaluation (GRADE) evidence profile (Table 2). As per the GRADE system, evidence from RCTs was considered to be strong evidence, which is down-rated if there are serious limitations, imprecision, inconsistency, indirectness or publication bias. Observational studies are rated as low quality and up-rated if the magnitude of the effect is large, has minimal confounders and there is a dose-dependent effect [12].

\section{Data Synthesis and Analysis}

The evidence base was individually rated for different end points within a single study. Studies synthesizing the previous evidence base along with the expert opinions were also considered as an evidence base. The efficacy end points considered in pre-clinical studies included reduction in viral copy numbers evaluated by real-time polymerase chain reaction (RTpolymerase chain reaction [PCR]) and lack of viral nucleoprotein assessed by immunofluorescence. However, in case of clinical studies, the various end points of efficacy assessed were either clinical cure (time to body temperature normalization, duration of cough, death or clinical worsening of disease) or virologic cure (negative RT-PCR).

\section{RESULTS}

\section{Pharmacologic Interventions}

Based on the systematic review of published evidence, the different pharmacologic interventions explored for the therapeutic management of patients with COVID-19 were chloroquine/hydroxychloroquine, remdesivir, arbidol, lopinavir, ritonavir, glucocorticoids, immune modulators, immunoglobulin/plasma therapy, tissue plasminogen activator, recombinant erythropoietin, tocilizumab, baricitinib, ivermectin, tetracyclines, statins, homoharringtonine and metronidazole. All the drugs that have been explored as therapeutic options were previously used for the treatment of other clinical conditions. Hence, the evidence base does not follow the conventional pre-clinicalearly clinical (phases I and II) phase III studies. On the contrary, the drugs are repurposed, and hence the main aims of later-stage clinical trials are to reposition the drug for COVID-19 (repositioning clinical trials) [13].

\section{Chloroquine and Hydroxychloroquine}

Chloroquine is a 9-aminoquinoline, which is a weak base and facilitates an antimicrobial effect by increasing the $\mathrm{pH}$ of acidic vesicles. It has been safely used for the treatment of malaria, amoebiasis and autoimmune diseases [14]. The first evidence of its activity against $\mathrm{CoV}$ was provided by Vincent et al. in Vero E6 cells against SARS-CoV. They confirmed the prophylactic effect of chloroquine in Vero E6 cells that were pretreated with $10 \mu \mathrm{M}$ of chloroquine, which reduced the infectivity by $100 \%$ compared with the control. Similarly, the addition of $0.1-1 \mu \mathrm{M}$ of chloroquine after infection reduced the infection by $50 \%$, suggesting the probable therapeutic effect of chloroquine in SARS-CoV infection [14]. The anti-SARS-CoV-2 activity of chloroquine was assessed by Wang et al. in the Vero E6 cell line. The time-of-addition assay suggested a probable role of chloroquine at the entry and post-entry stages of SARS-CoV-2 infection. The effective concentration $\left(\mathrm{EC}_{90}\right)$ was found to be $6.90 \mu \mathrm{M}$, which is clinically achievable with the administration of 500-mg chloroquine [15]. In pharmacokinetic modeling studies, hydroxychloroquine, which is an analog of chloroquine, was found to be more potent than chloroquine with a better safety profile [16].

The first clinical evidence of efficacy was reported by Gautret et al. from a cohort of French patients who were treated with $600 \mathrm{mg}$ of hydroxychloroquine. The study included 42 patients (26 patients treated with hydroxychloroquine and 16 patients in the control group) who were confirmed to be positive for 


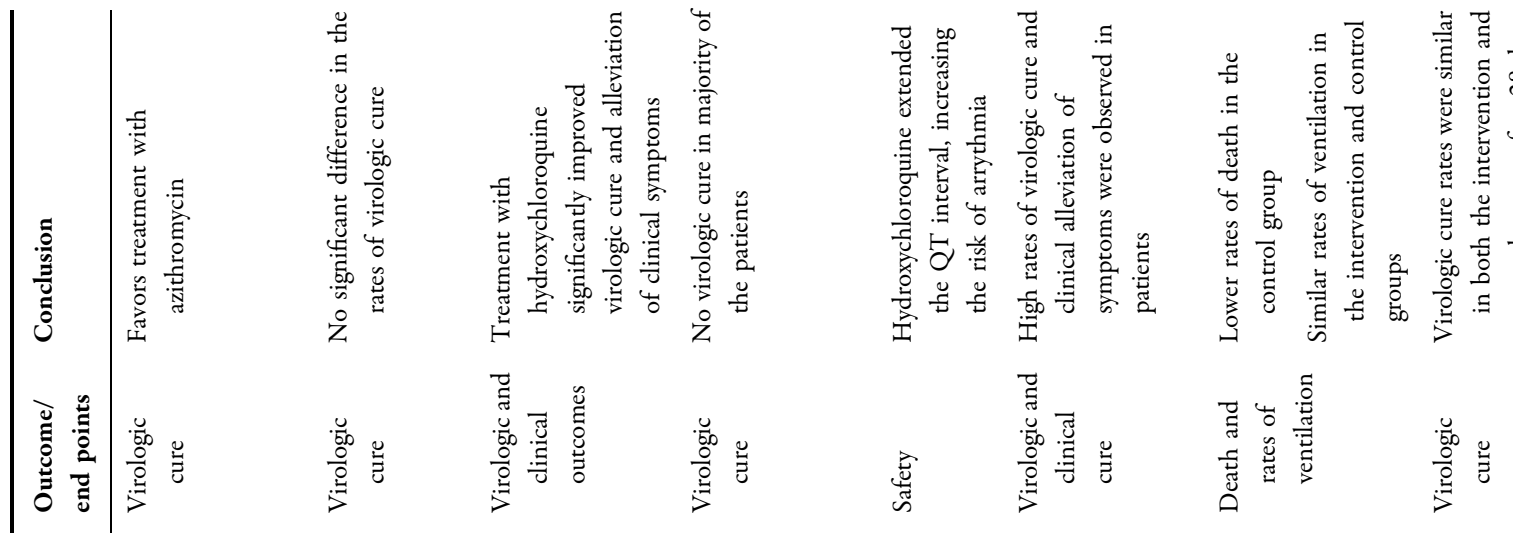

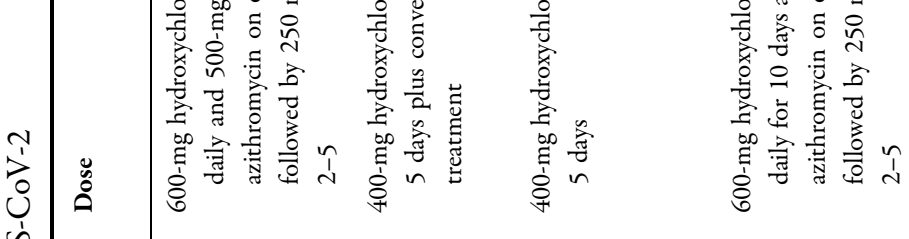

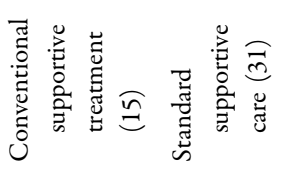
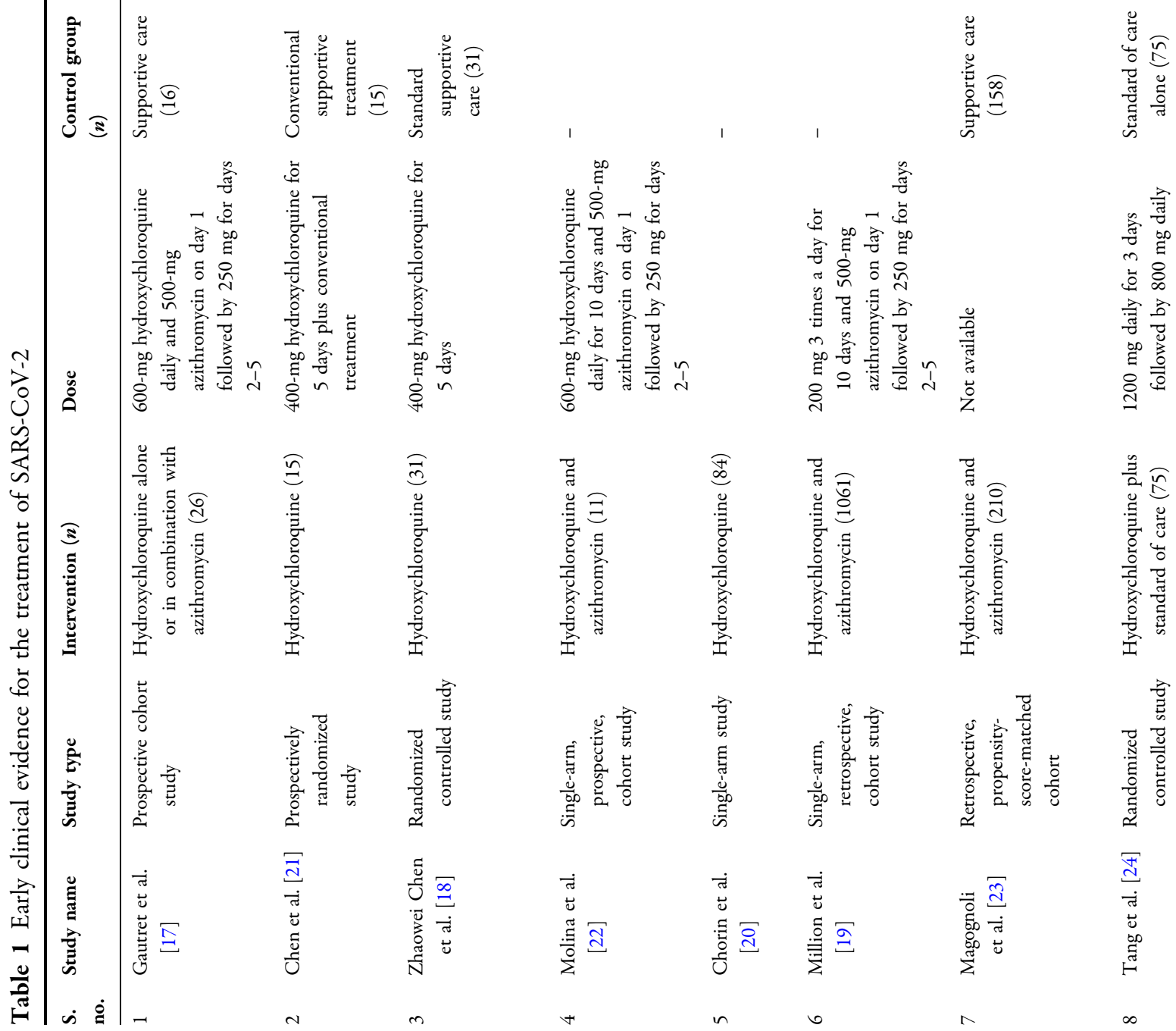

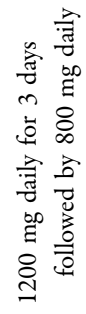
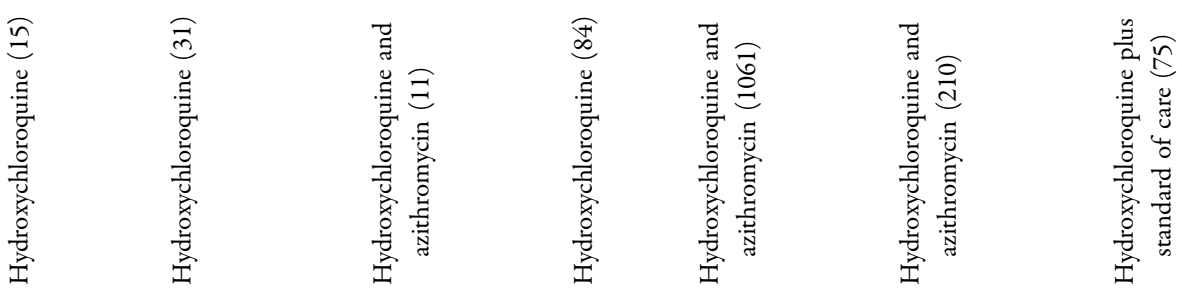

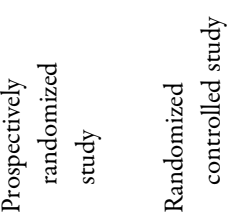
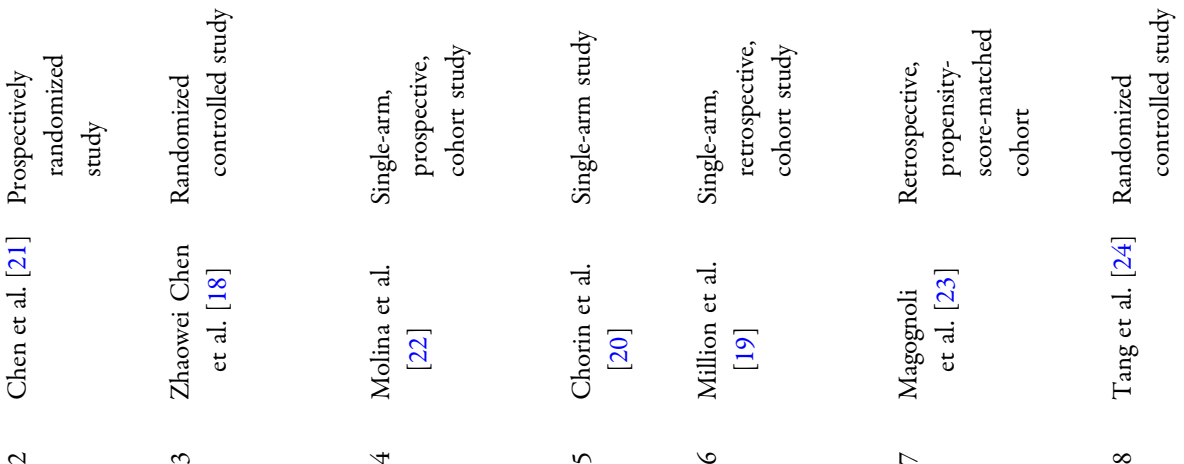


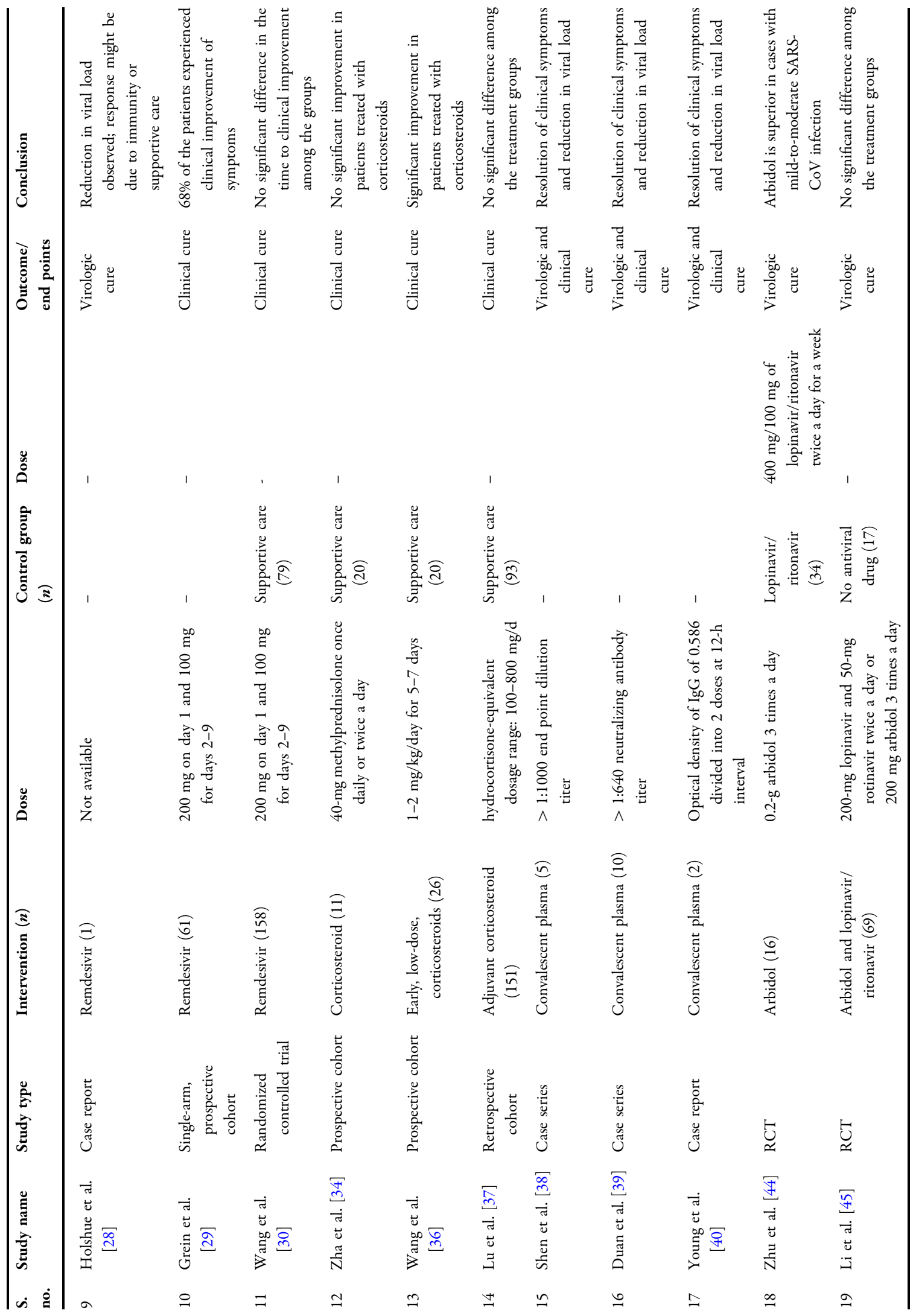




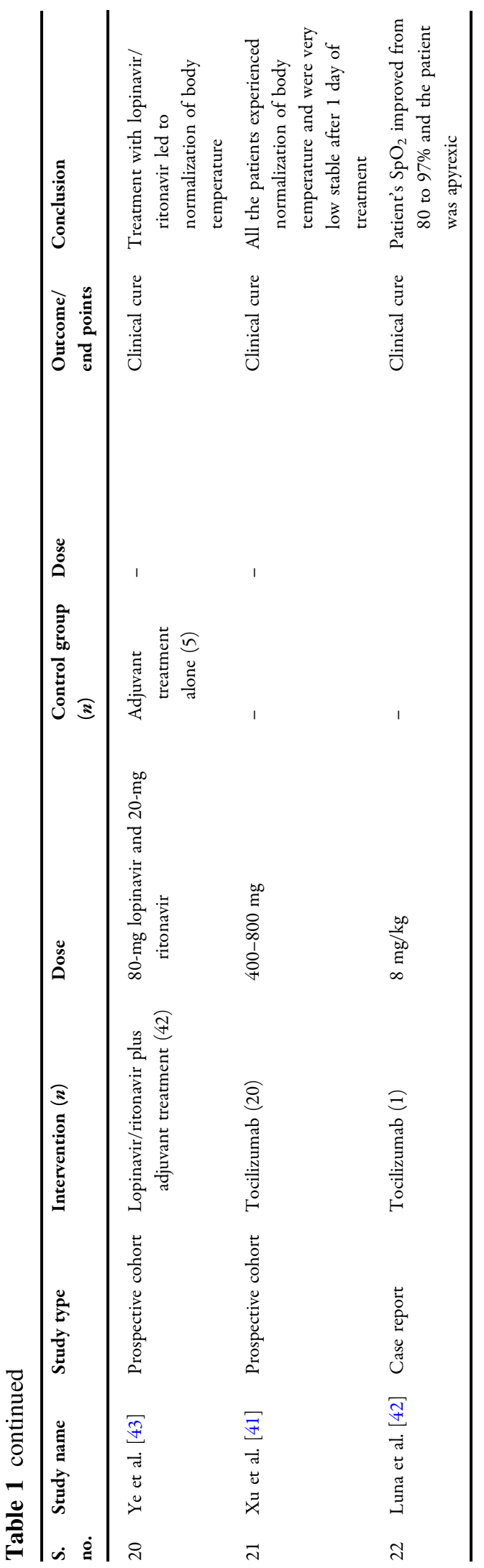

SARS-CoV-2 by RT-PCR. Of the 20 patients treated with hydroxychloroquine available for efficacy assessment, 14 (70\%) patients experienced virologic cure after 6 days of treatment, whereas only $2(12.5 \%)$ patients in the control group were negative for SARS-CoV-2 after 6 days of treatment. A subgroup of patients in the hydroxychloroquine group was also treated with azithromycin (6 patients), and all of them experienced virologic cure, suggesting a better efficacy for hydroxychloroquine in combination with azithromycin than hydroxychloroquine alone (100\% vs. $57 \%$, respectively) [17].

The first evidence of the efficacy of hydroxychloroquine from an RCT was published recently in the preprint server MedRxiv. The study recruited 62 patients positive for SARSCoV-2 and randomly divided them into the test (hydroxychloroquine) and control (placebo) groups. Comparison of radiologic findings revealed that $61.3 \%$ of the patients in the hydroxychloroquine group showed significant improvement, whereas only $16.1 \%$ of those in the control group had significant improvement. The body temperature recovery time was also significantly reduced in the hydroxychloroquine group (2.2 [0.4] days) compared with the control group (3.2 [1.3] days). Similarly, cough remission time was also significantly reduced in the hydroxychloroquine group [18].

The efficacy of hydroxychloroquine in combination with azithromycin was also reported in a retrospective study involving French patients. A total of 1061 SARS-CoV-2-positive patients treated with hydroxychloroquine (200 mg 3 times a day) in combination with azithromycin were included in the study (500 $\mathrm{mg}$ on day 1 followed by $250 \mathrm{mg}$ daily for the next 4 days). Virologic cure and clinical outcomes were assessed. Approximately $92 \%$ of the patients experienced virologic cure (viral culture and RTPCR), and $95 \%$ of the patients reported alleviation of clinical symptoms. Multivariate analysis revealed older age (odds ratio [OR] 1.11, 95\% confidence interval [CI] 1.07-1.15), selective beta-blocking agents (OR 4.16, 95\% CI 1.19-14.55), angiotensin II receptor blockers (OR 18.40, 95\% CI 6.28-53.90) and medium and high national Early Warning Score (NEWS; OR 9.48, 95\% CI 3.25-27.66; OR = 10.05, 95\% 


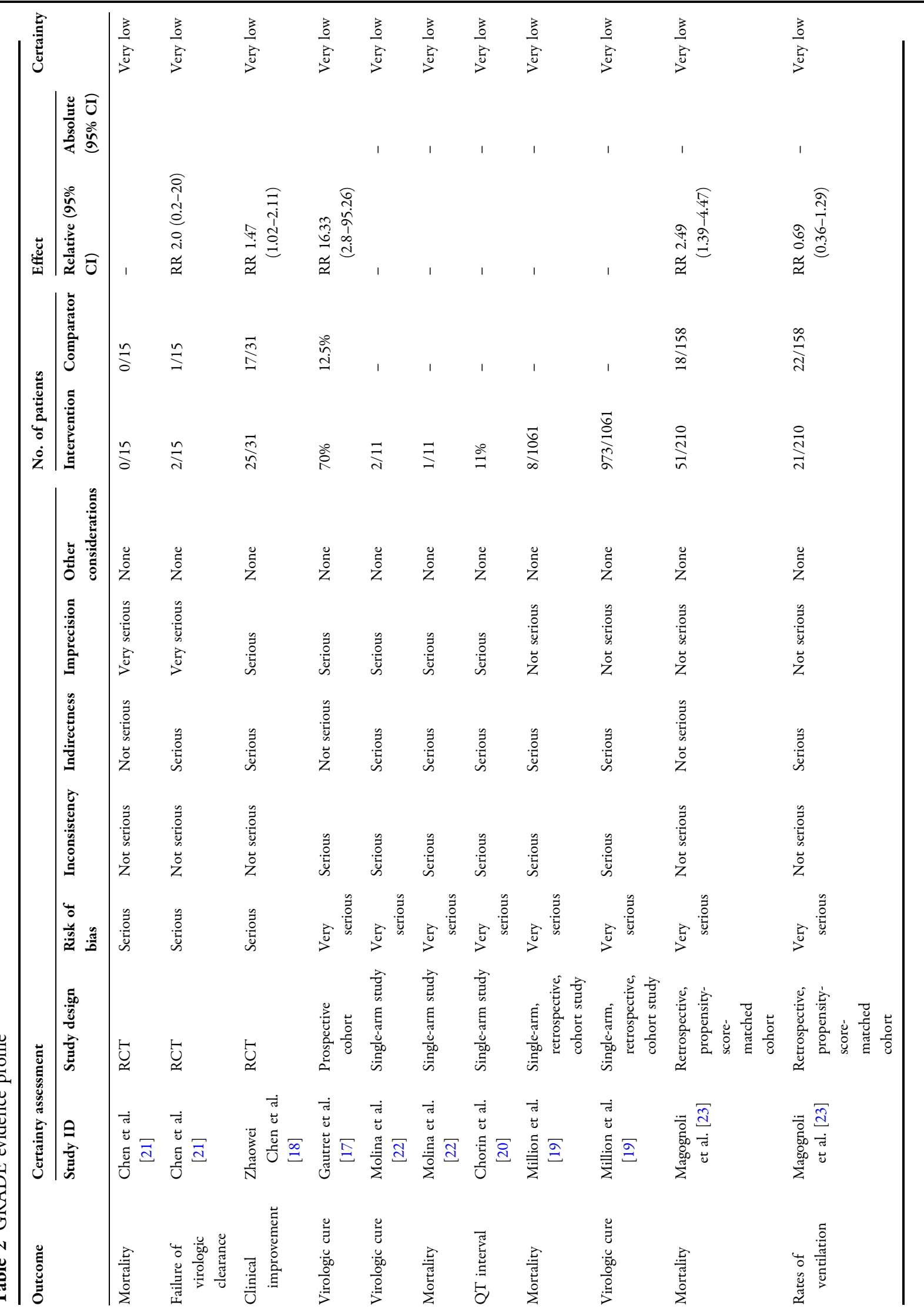




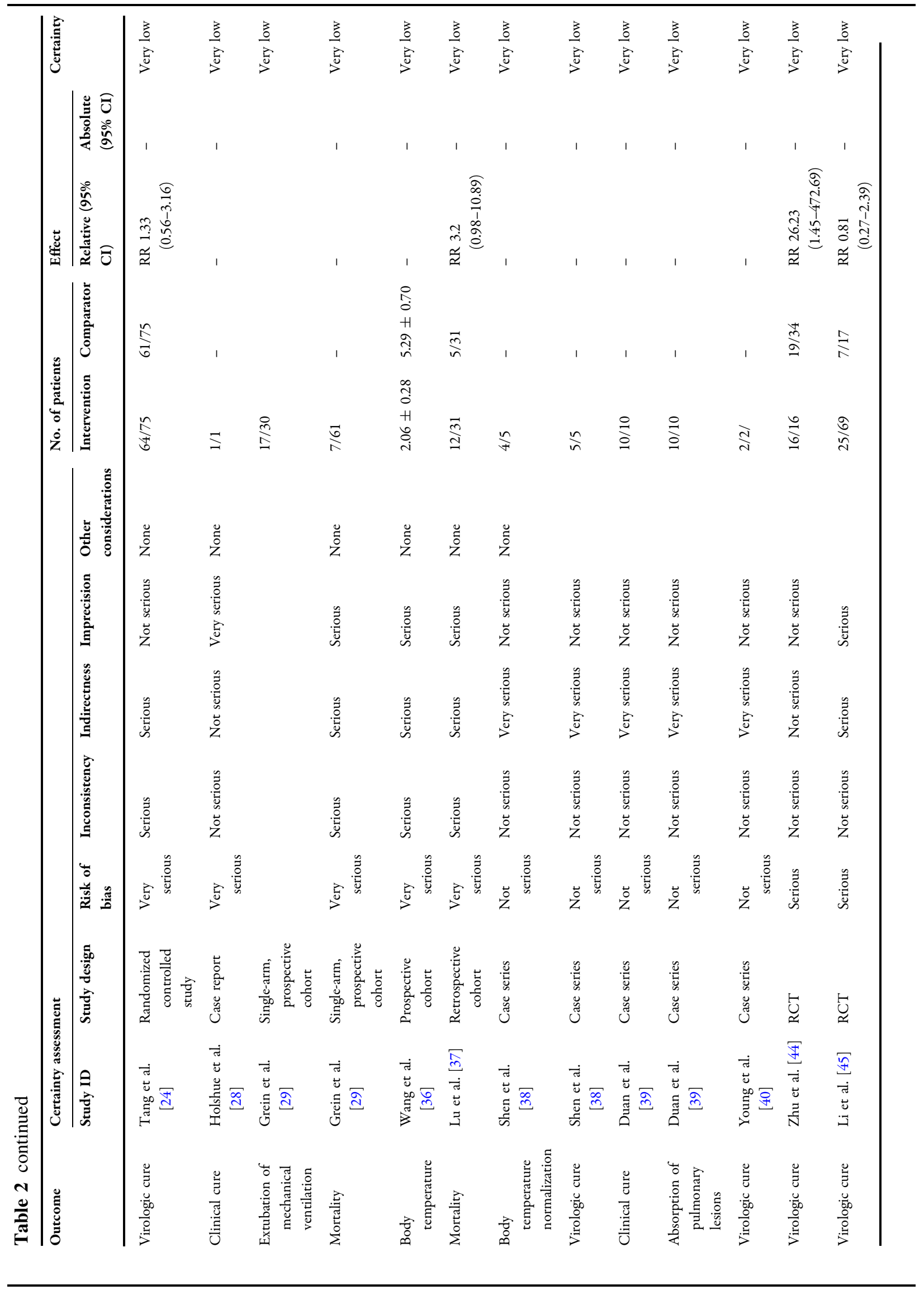




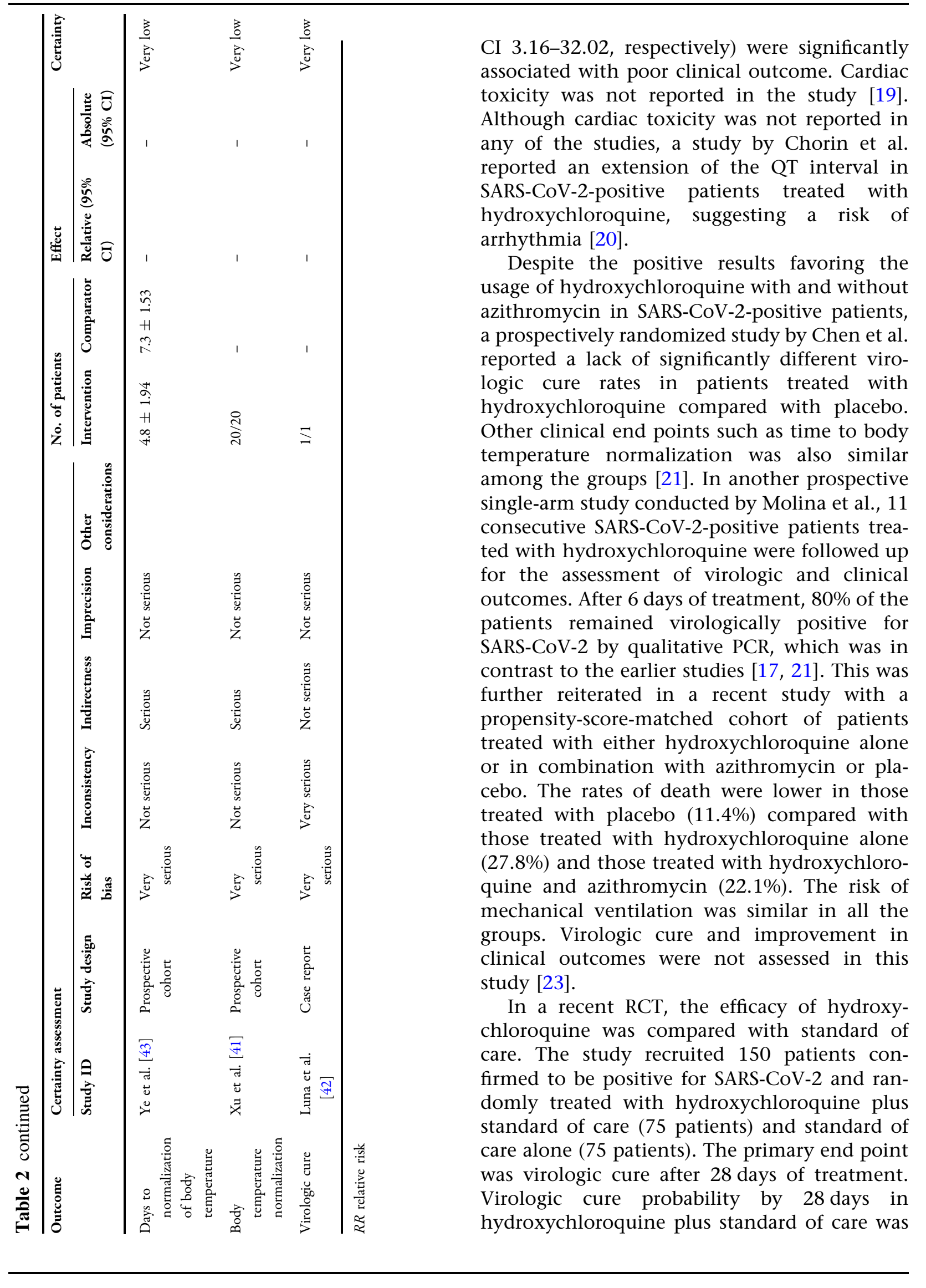


$85.4 \% \quad(95 \%$ confidence interval [CI] 73.8-93.8\%), which was similar to that in the standard of care alone group (81.3\%; 95\% CI 71.2-89.6\%) [24]. A summary of the available evidence for hydroxychloroquine is provided in Table 1.

Currently available early clinical evidence provides contradictory findings on the efficacy of hydroxychloroquine in SARS-CoV-2-positive patients. There are currently 125 clinical trials registered in the WHO International Clinical Trials Registry Platform (ICTRP; https:// clinicaltrials.gov/ct2/who_table) and 192 studies registered in clinicaltrials.gov. The results of the ongoing studies will provide conclusive evidence on the efficacy of hydroxychloroquine in the treatment of SARS-CoV-2.

\section{Remdesivir}

Remdesivir is a nucleoside analog with proven activity against RNA viruses causing lethal hemorrhagic fever (Nipah and Ebola). It is an RNA-dependent RNA polymerase inhibitor capable of inhibiting multiple CoVs [25]. In a mouse SARS virus experimental model, the administration of remdesivir 1 day after the infection reduced the virus titer in the lungs. Similar findings were also observed with a rhesus monkey model of MERV-CoV [25-27]. The pre-clinical efficacy of remdesivir was confirmed in in vitro studies with Vero E6 cell lines [15].

The probable therapeutic effect of remdesivir in a patient with SARS-CoV-2 was initially reported in a case report wherein remdesivir was used on compassionate grounds. Although virologic improvement and clinical cure were observed in the patient, remdesivir was used only on the 6th day of admission, and the continuous viral load testing revealed that a reduction in viral load had begun before the administration of the drug. Hence, the observed clinical effect might be due to immunity and supportive treatment [28]. A larger prospective cohort of patients treated with remdesivir on compassionate grounds was recently reported by Grein et al. [29]. Of the 61 patients who received at least one dose of remdesivir, 53 patients were available for follow-up. After a median follow-up of 18 days, 36 patients (68\%) had an improvement in oxygen-support class, including 17 of 30 patients (57\%) receiving mechanical ventilation. A total of 25 patients (47\%) were discharged, and 7 patients (13\%) died with a mortality of $18 \%$ (6 of 34) among patients receiving invasive ventilation and 5\% (1 of 19) among those not receiving invasive ventilation [29].

The efficacy of remdesivir was recently evaluated in an RCT involving Chinese patients compared with placebo. The study enrolled 237 patients (158 to remdesivir and 79 to placebo) and evaluated the clinical improvement up to day 28. The results of the study revealed a lack of significant difference in time to clinical improvement in patients treated with remdesivir compared with placebo (hazard ratio, 1.23 [95\% CI 0.87-1.75]). In patients with symptoms for $\leq 10$ days, remdesivir showed better efficacy (hazard ratio (HR), 1.52 [95\% CI 0.95-2.43]), albeit without statistical significance [30].

The early clinical evidence for the efficacy of remdesivir is inconclusive with only marginal efficacy. But owing to the differences in the end points considered in the studies, the precise role of remdesivir may require larger studies with the assessment of both virologic and clinical outcomes. At present, there are 22 trials registered in clinicaltrials.gov and 13 studies registered in the WHO-ICTRP.

\section{Corticosteroids}

Corticosteroids are immune modulators that suppress the inflammatory response, thereby minimizing tissue damage. The early observational evidence for the effective use of corticosteroids stems from the lower prevalence of SARS-CoV-2 infection in patients with chronic respiratory disease, suggesting a role for the drugs given for chronic respiratory disease in reducing the prevalence of SARS-CoV-2 infections in such patients [31]. The early pre-clinical evidence provided by Matsuyama et al. reported effective antiviral activity of ciclesonide in inhibiting the replication of SARS-CoV-2 in epithelial cell lines with an effective concentration of $6.3 \mu \mathrm{M}$ [32]. Despite the anti- 
inflammatory effect provided by corticosteroids, they also cause immune suppression, delaying viral clearance [33].

A recent prospective cohort study conducted by Zha et al. recruited 31 SARS-CoV-2-positive patients treated with corticosteroids (11 patients) or supportive care. The study found no statistically significant association between treatment with corticosteroids and virus clearance time (HR 1.26; 95\% CI 0.58-2.74), hospital length of stay (HR 0.77 ; 95\% CI $0.33-1.78$ ) or duration of symptoms (HR 0.86; 95\% CI 0.40-1.83) [34]. A recent meta-analysis also suggested a higher relative risk for mortality and longer length of stay in patients with SARS-CoV and MERS-CoV treated with corticosteroids [35].

In a retrospective cohort study conducted by Wang et al., 46 SARS-CoV-2-positive patients treated with either corticosteroids [25] or supportive care [19] were analyzed for clinical outcomes. The mean duration for body temperature back to the normal range was significantly shorter in patients treated with corticosteroids compared with those without the administration of corticosteroids $(2.06 \pm 0.28$ vs. $5.29 \pm 0.70 ; P=0.010$ ). The patients included in the study had severe pneumonia and were treated early with a low dose of corticosteroid, suggesting a favorable effect of early, low-dose treatment [36]. On the contrary, another observational study conducted by Lu et al. reported a limited effect of adjuvant treatment with corticosteroids in critically ill patients [37].

The early clinical evidence for the treatment with corticosteroids remains inconclusive. There are 72 RCTs currently under progress to evaluate the efficacy of different corticosteroids at different stages of SARS-CoV-2 infection (clinicaltrials.gov).

\section{Immunotherapy with Convalescent Plasma/Sera}

The early evidence of the efficacy of convalescent plasma/sera was provided by two case series from China. In the first study, five critically ill patients with acute respiratory distress syndrome (ARDS) were administered convalescent plasma/sera containing neutralizing IgG antibody at a titer of $>1: 1000$ that had been obtained from five patients previously recovered from SARS-CoV-2. All the patients were on mechanical ventilation at the time of treatment and previously treated with antiviral agents and methylprednisolone. After the treatment with convalescent plasm/sera, body temperature normalized after 3 days in four of the five patients, and viral loads also became negative after 12 days of treatment. ARDS was resolved in four patients after 12 days of treatment, and three patients were discharged [38]. In another prospective case series, ten patients were treated with convalescent plasma/sera with a neutralizing antibody titer of $>1: 640$. The radiologic examination revealed the resolution of lung lesions after 7 days and virologic cure in seven patients [39]. These findings were also substantiated by a case report of two elderly patients treated with convalescent plasma/sera from South Korea. Both the patients had been previously treated with hydroxychloroquine and lopinavir/ritonavir. Both the patients experienced virologic cure after 3 days of treatment with convalescent plasma/sera. The resolution of lung lesions was also observed along with alleviation of other clinical symptoms [40]. Although the evidence base for immunotherapy with convalescence plasma/sera is supported by only weak quality evidence, it holds promise for future management strategies.

\section{Tocilizumab}

Previous studies on MERV-CoV and SARS-CoV-1 have revealed the release of a plethora of cytokines, including IL-6, which was also confirmed in SARS-CoV-2 infection [41]. Hence, tocilizumab, which is a monoclonal antibody targeting IL-6, was explored as a treatment option in the treatment of cases with severe SARS-CoV-2 infection. The earliest evidence of its efficacy was provided from a case series by $\mathrm{Xu}$ et al. The study included 21 patients (17 severe and 4 critical) who were treated with tocilizumab. Irrespective of the disease severity, all the patients experienced normalization of body 
temperature 1 day after the treatment with tocilizumab. The oxygen saturation $\left(\mathrm{SpO}_{2}\right)$ levels were also improved significantly, and one-third of patients on ventilator support were put on a noninvasive ventilator a day after the treatment. The percentage of lymphocytes and C-reactive proteins also returned to normal in most patients after 5 days of treatment [41].

In a subsequent case report, tocilizumab was also used successfully for treating a patient with sickle cell anemia [42]. The patient was hospitalized and, on day 1, developed symptoms of SARS-CoV-2 infection, including fever $\left(38.5^{\circ} \mathrm{C}\right)$ and $\mathrm{SpO} 2$ dropping to $91 \%$ with crackles at pulmonary auscultation. The patient was treated with antibiotics and hydroxychloroquine at a dosage of $200 \mathrm{mg}$ orally every $8 \mathrm{~h}$ while the results of RT-PCR were awaited. The patient was treated with 1 pulse of intravenous tocilizumab at a dosage of $8 \mathrm{mg} / \mathrm{kg}$ on day 2 after the deterioration of symptoms and had a positive result in RT-PCR for SARS-CoV-2 infection. The patient experienced clinical cure with an improvement in $\mathrm{SpO} 2$ and was discharged on day 5 [42]. The confounding effect of early hydroxychloroquine treatment cannot be ruled out as a possible cause of early clinical cure in this patient. Further clinical studies are required to substantiate the utility of tocilizumab in the treatment of SARS-CoV-2 patients.

\section{Other Antiviral Drugs}

Among the antiviral drugs, lopinavir, ritonavir and arbidol were explored in clinical studies involving SARS-CoV-positive patients. In a prospective cohort study conducted by Ye et al., 47 patients treated with either lopinavir/ritonavir or adjuvant treatment were analyzed for efficacy outcomes. The study reported favorable outcomes with respect to lowering body temperature in patients treated with lopinavir/ritonavir compared with adjuvant treatment alone [43].

In a recent RCT, 50 patients with laboratoryconfirmed COVID-19 were treated either with lopinavir/ritonavir (34 cases) or arbidol (16 cases). All the patients had mild-to-moderate SARS-CoV-2 infection without ARDS. The reduction in viral load was the primary end point. After 14 days of treatment, virologic cure was observed in all the patients treated with arbidol, but $15(44.1 \%)$ patients treated with lopinavir/ritonavir still had detectable viral load. The study concluded the superior effect of arbidol over lopinavir/ritonavir in the treatment of cases with mild-to-moderate SARS-CoV infection [44]. In another RCT, 86 patients with mild-to-moderate SARS-CoV-2 infection were randomly assigned to the lopinavir/ritonavir group [33] or the arbidol group [34] or the no antiviral drug group [16] [16]. The primary end point was virologic cure. The study reported similar rates and duration of virologic cure in all three groups, suggesting lack of clinical efficacy of antiviral drugs in the treatment of cases with mild-to-moderate SARS-CoV-2 infection [45].

\section{Early Evidence and Treatment Guidelines}

The treatment guidelines provided by different nodal governmental agencies are evidencebased and are subject to change based on the availability of newer evidence. They also take into account the local availability of drugs and evidence from studies done in specific geographies [46]. Considering the evidence-based nature of treatment guidelines and their implications for patient care, we have included the different treatment guidelines and have also summarized the changes they have gone through mainly because of the evolution of the clinical evidence landscape. Early treatment guidelines were limited by their methodologic restrictions arising from the lack of quality clinical evidence.

Despite the poor quality of early evidence, multiple treatment options were recommended by different nodal agencies. WHO had published guidance document for the therapeutic management of COVID-19 patients in March 2020, which was updated in May 2020. The early treatment guideline released in March did not recommend any pharmacologic intervention except symptomatic management of COVID-19 patients [47]. As per the recent guideline document from WHO, chloroquine/ hydroxychloroquine, lopinavir/ritonavir, 
remdesivir, unifenovir, favipiravir, tocilizumab and plasma therapy are recommended only in the context of clinical trials and glucocorticoids are recommended in any treatment setting [48]. Among the treatment options, hydroxychloroquine and chloroquine were provided emergency use authorization by the Food and Drug Administration (FDA) for the treatment of COVID-19 patients based on early evidence. However, based on the subsequent clinical evidence the early use authorizations provided for chloroquine and hydroxychloroquine were revoked as the adverse events outweighed the potential benefits [49]. Similarly, remdesivir was also given emergency use authorization by the FDA [50].

As per the National Institutes of Health (NIH) guidelines, chloroquine and hydroxychloroquine (with and without azithromycin) are not recommended for the treatment of COVID-19 patients except in clinical trial setting. With respect to remdesivir, the NIH guidelines recommends its use in hospitalized patients, and it should be used on priority to treat patients requiring supplemental oxygen. Furthermore, lopinavir and ritonavir were also not recommended for routine use in COVID-19 patients except in the context of clinical trials. The evidence base for convalescent plasma/sera is also not sufficient to recommend in favor of or against their use in the treatment of COVID19 patients. Based on the recently published RECOVERY trial, NIH recommends the usage of dexamethasone in patients who either require supplemental oxygen or are on mechanical ventilation [51].

As per the updated guidelines from the Infectious Diseases Society of America, hydroxychloroquine, chloroquine and azithromycin are recommended only in the setting of a clinical trial with a conditional recommendation with low certainty evidence. Similarly, lopinavir/ritonavir, convalescent sera and tocilizumab are also recommended only in the context of clinical trials, whereas corticosteroids are recommended only for severe COVID-19 patients in routine hospital settings. Corticosteroids are not recommended for the treatment of patients with mild-to-moderate SARS-CoV-2. Remdesivir is also recommended for treatment in patients with severe COVID-19 routine hospital settings [52].

The treatment guidelines released by the Chinese National Health Commission have also undergone extensive evidence-based changes, and the recent 7 th edition was published in March 2020. It recommended the use of $\alpha$-interferon atomization inhalation (5 million units per time for adults in sterile injection water, twice a day) and lopinavir/ritonavir orally, two capsules each time twice a day, based on weak evidence. It also recommended $40-80 \mathrm{mg}$ /day methylprednisolone based on weak evidence and tocilizumab, convalescent plasma/sera therapy and glucocorticoids were also recommended for treatment [53].

The Directorate of Health Services, Government of India, had released multiple updated guideline documents pertaining to different aspects of COVID-19 including therapeutic management. As per the early management guidelines released in March, off-label use of hydroxychloroquine and azithromycin were recommended without any mention about specific target patient groups [54]. Hydroxychloroquine was also recommended as chemoprophylaxis in high-risk healthcare workers and asymptomatic first-degree contacts as per an advisory released in March 2020. This recommendation was based on pre-clinical data, and the advisory was updated in May 2020 providing observational evidence on the lower incidence of COVID-19 in healthcare workers who were on prophylactic treatment with hydroxychloroquine [55]. As per the recent evidencebased guidelines released in July, off-label use of hydroxychloroquine should be used after discussing the potential implications with patients and should be used in an early stage of disease. The available evidence is not sufficient to recommend against the use of hydroxychloroquine. Remdesivir was provided emergency use authorization and may be used in patients requiring supplemental oxygen. Convalescent plasma/sera could be considered in patients with progressive requirement for supplemental oxygen depending on the availability (4 to $13 \mathrm{ml} / \mathrm{kg}$, usually $200 \mathrm{ml}$ single dose given slowly over not less than $2 \mathrm{~h}$ ). The guidelines also recommend off-label use of tocilizumab in 
mechanically ventilated patients. Unlike other guidelines, both the early and recent Indian guidelines recommend the use of glucocorticoids including dexamethasone in patients with progressive deterioration of oxygenation indicators [56].

Apart from the above guidelines, multiple national guidelines have been released by the affected countries based on available evidence, consensus and local availability. Accordingly Russian guidelines recommend lopinavir/ritonavir, recombinant interferon and ribavirin in moderate-to-severe infections; French guidelines recommend lopinavir/ritonavir and hydroxychloroquine; Dutch guidelines recommend chloroquine, lopinavir/ritonavir and a combination of chloroquine with remdesivir and lopinavir/ritonavir in severe disease [57]. The evidence base for most of these recommendations is not strong, and most of these guidelines did not grade the evidence based on the GRADE rating.

\section{Recent Evidence}

Apart from the early clinical evidence, two RCTs evaluating dexamethasone and remdesivir were published recently, after our literature search time period. But we have included the trials as they also constitute an evidence base for recent therapeutic management guidelines. Since most of the early studies did not use all-cause mortality as a composite end point, the RECOVERY trial utilized all-cause mortality to evaluate the efficacy of dexamethasone, a glucocorticoid, compared to standard of care. Glucocorticoids are not recommended by multiple therapeutic management guidelines owing to lack of evidence and the profound immunosuppressive effects [57]. The RECOVERY trial randomized a total of 6425 patients to receive either dexamethasone $(n=2104)$ or standard of care $(n=4321)$. The rates of all-cause mortality were significantly lower in the dexamethasone arm compared to standard of care (22.9 vs. 25.7; rate ratio: $0.83 ; P<0.0001)$. The study recruited patients with different baseline severity levels, and a prespecified subgroup analysis revealed significant reduction in all-cause mortality only in patients requiring respiratory support at randomization. This is in line with the anti-inflammatory activity of dexamethasone, which might be pronounced and beneficial only during specific stages of the disease. In early-stage SARS-CoV 2-positive patients, dexamethasone might alter the natural disease course [58].

Recently, the preliminary results of the Adaptive Covid-19 Treatment Trial (ACTT-1) that evaluated the efficacy of remdesivir in the treatment of SARS-CoV was published. The trial randomized 1063 patients to receive either remdesivir $(n=541)$ or placebo $(n=522)$ and evaluated the time to recovery with an eightstage ordinal scale based on the clinical condition at randomization. The overall rate ratio for recovery after adjusting for baseline ordinal scores was found to significantly favor remdesivir with an effect estimate of 1.31 $(P<0.0001)$. Across different ordinal score categories, a significant recovery rate ratio was observed only for patients with an ordinal score of 5 (hospitalized, requiring any supplemental oxygen). The HR for mortality was also significantly favoring the remdesivir group only for patients with an ordinal score of 5 (HR 0.22 , 95\% CI 0.08, 0.58). However, in the overall population, there was no significant reduction in mortality in the remdesivir arm (HR 0.70; $95 \%$ CI $0.47,1.04)[59]$.

\section{Perspectives on Early Clinical Evidence}

The normal workflow in the development of treatment options for any indication involves pre-clinical to early clinical to late stage clinical trials. However, the normal workflow is hampered in case of pandemics. Based on the early evidence, the therapeutic options available were drugs attenuating either the immunologic response (anti-inflammatory drugs) or the viral load (antiviral agents). Since the pathogenesis of SARS-CoV-2 involves mainly inflammatory responses, the most promising drugs are antiinflammatory drugs. The inflammatory response is also related to the viral load, which makes the selection of the ideal end point difficult. From the early evidence and from the trials in the pipeline, the most common end 


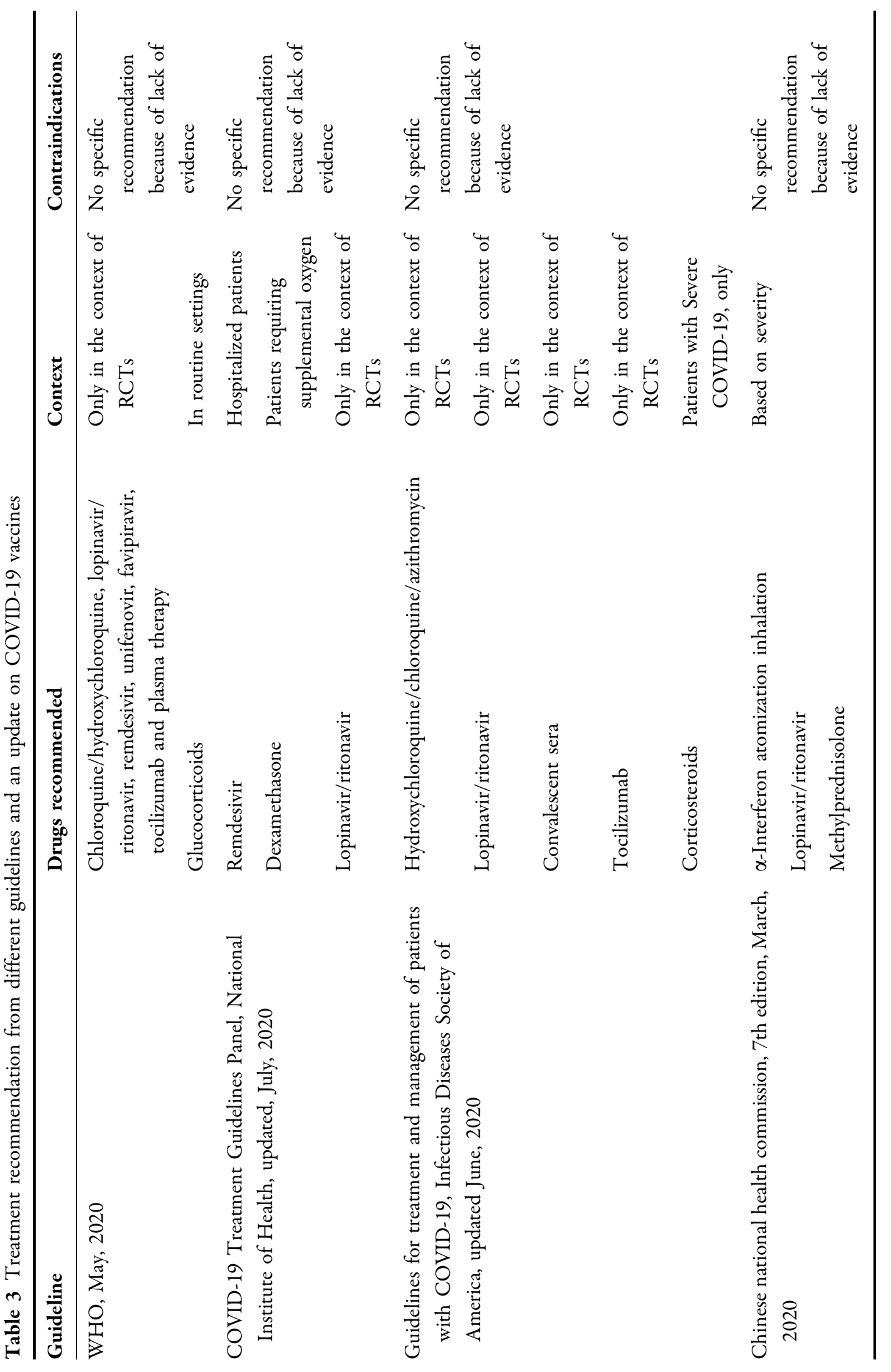




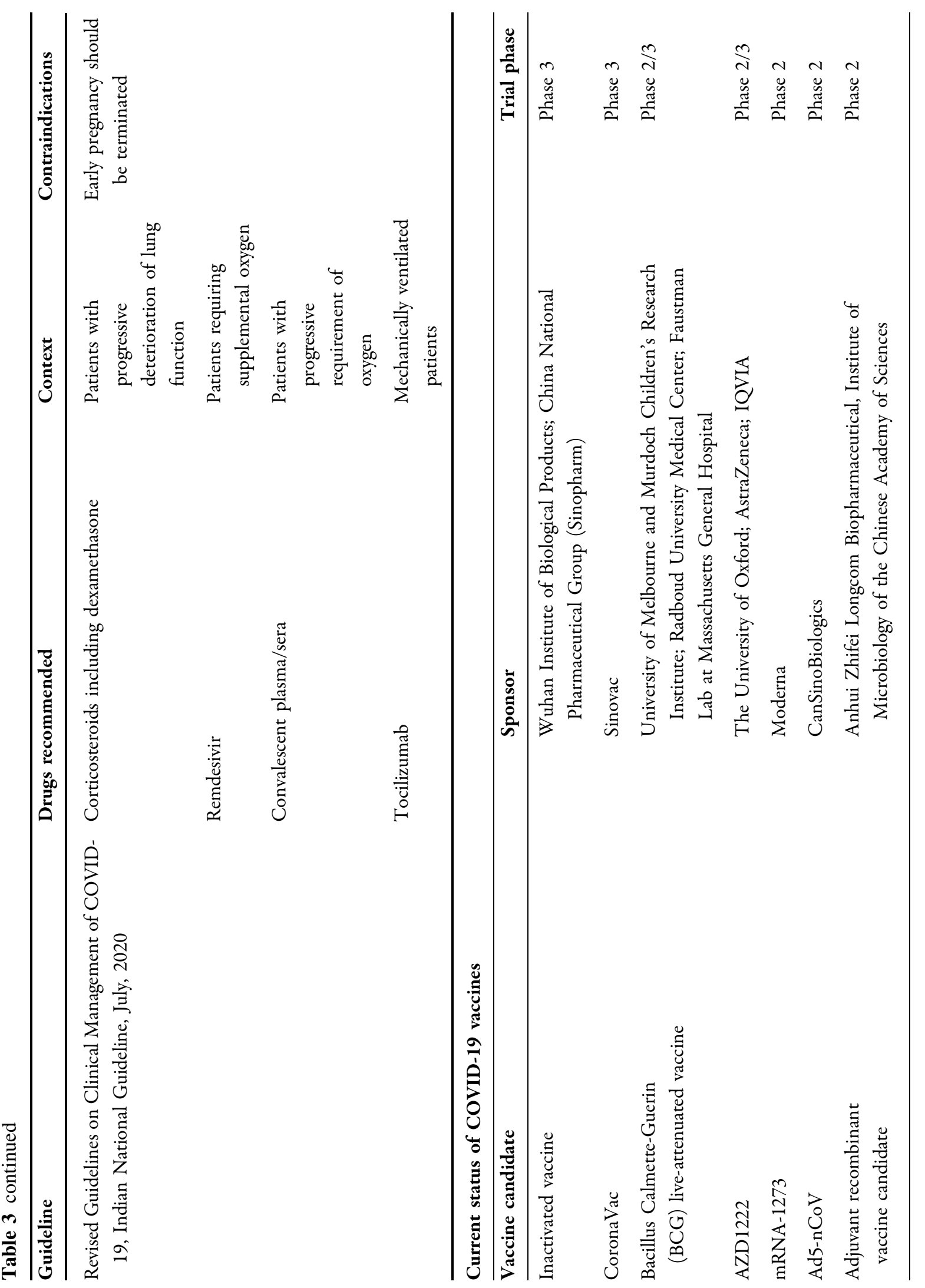




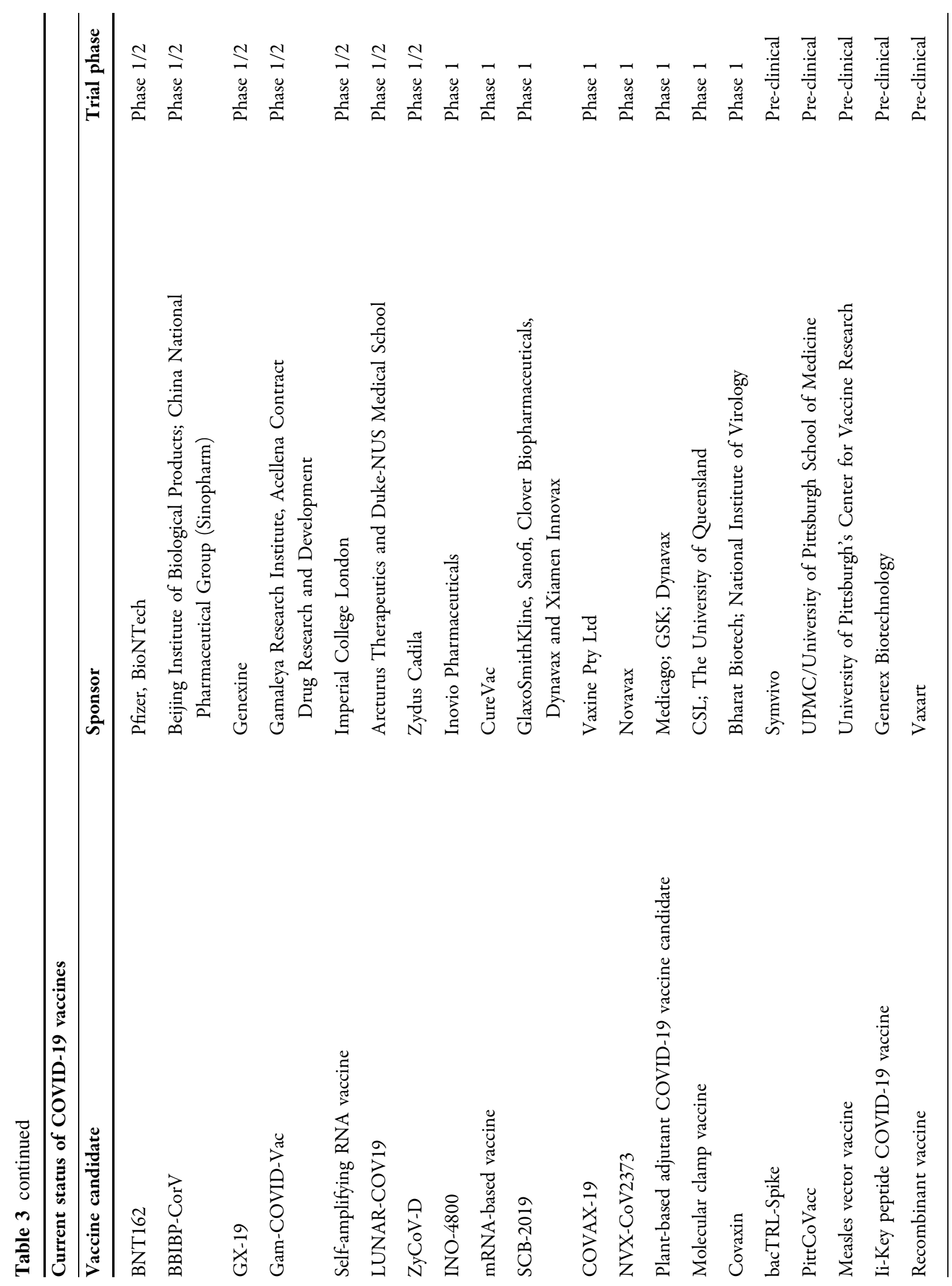




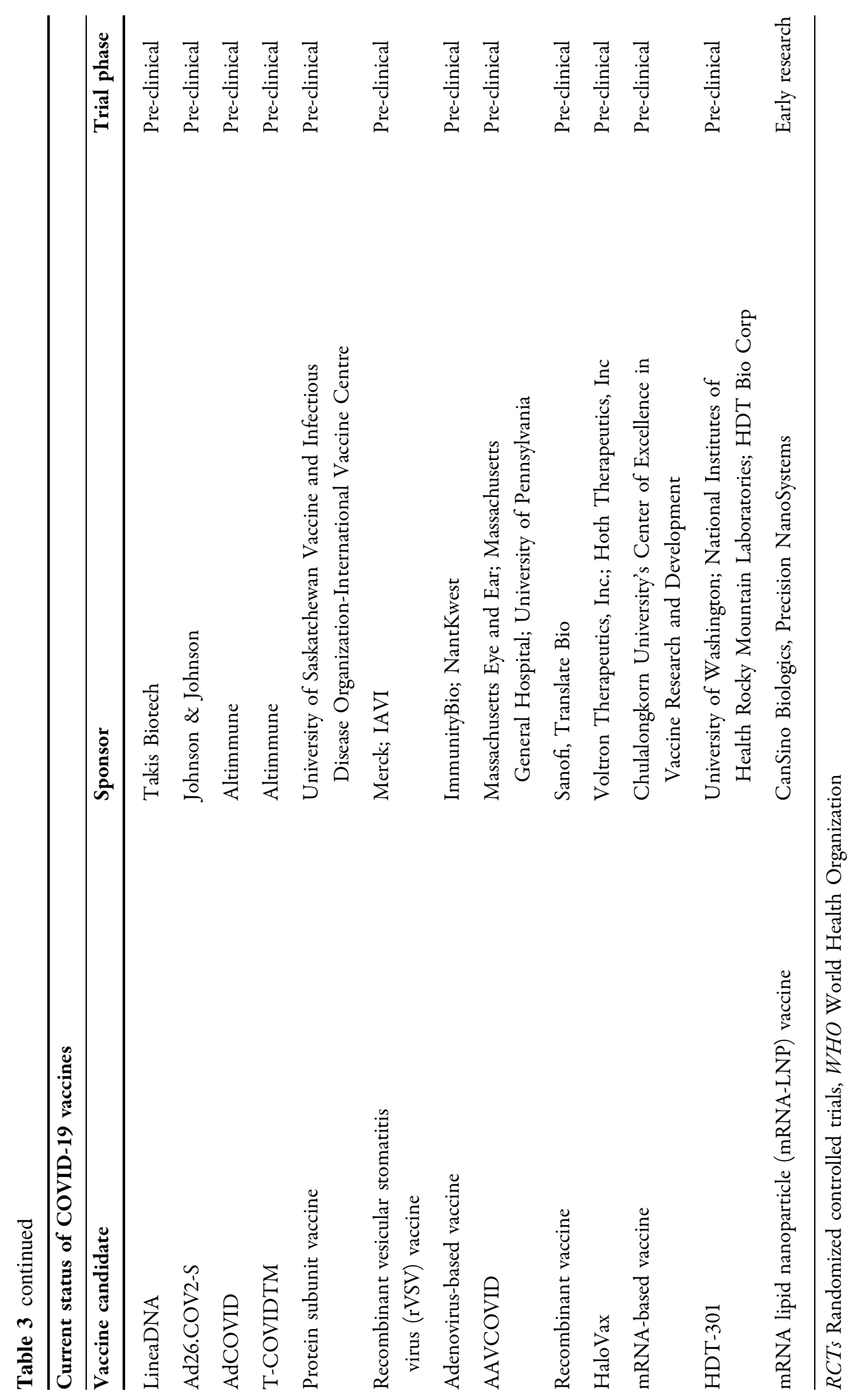


points assessed were a 6- or a 7-point ordinal scale outcome that defines clinical benefit based on the baseline clinical condition till the end of the study or death, whichever is earlier. Depending on the study, a 2- or 3-point decrease in the selected ordinal scale was considered a marker of clinical efficacy. However, overall mortality might be the ideal composite end point that represents clinical benefit in a pandemic outbreak. On the contrary, overall mortality might not take into account the initial patient status, thereby confounding the results. In the prevailing scenario, ordinal-scale end points as a marker of clinical improvement and virologic cure might be robust indicators of clinical improvement, but a consensus on the ordinal scale with respect to patients with different severities of diseases might help in selecting the optimum therapeutic management strategy based on clinical evidence [60].

A case in point is the issue with the realworld evidence on the efficacy of hydroxychloroquine [61]. This was a multi-national registry-based study, which was among the earliest evidence in support of hydroxychloroquine for the treatment of SARS-CoV-2. However, subsequent clinical trials provided contradictory results that led to the questioning of the results of the study and subsequent journal retraction. In our systematic literature review, this study was also considered as this was early clinical evidence. The availability of preprint servers also plays a huge role in dissemination of early-stage evidence, which is a milieu never faced in the past. Although this leads to faster dissemination, it can also lead to propagation of junk science. We have also included evidence from preprint servers to make a comprehensive appraisal of early-stage evidence. Subsequent to the retraction of the Lancet study on hydroxychloroquine, the French study published in the preprint server MedRxiv was also withdrawn. Unlike the journal retractions, preprint retractions do not require substantial explanation of the reasons for retraction. This further complicates the quality of early evidence [62].

\section{Future Recommendations}

The early-stage evidence available does not provide convincing support in favor of or against a particular therapeutic regimen. This is mainly because of the heterogeneity with respect to the patients, pathogen variants and end points. Considering the fact that the last few pandemics were caused by respiratory viruses, drafting a consensus on the most appropriate end point will help in improving the quality of evidence in future pandemics. Furthermore, despite the availability of RCTs, they were of low quality because of the inherent bias (no data on blinding) and imprecision. Since the field of evidence-based medicine is a dynamically evolving field, future studies, especially studies of importance in dealing with medical emergencies, should be appropriately designed to provide reliable and timely evidence. Although the availability of preprint servers facilitates faster dissemination of data, the non-peer-reviewed nature of content needs to be interpreted with caution.

Vaccines hold great promise for the management and control of COVID 19. Currently, 42 vaccine candidates are under clinical development sponsored by academic, industry and governmental agencies. Out of 42 candidate vaccines, 24 vaccine candidates are in phase $1-3$ clinical development (Table 3) [63].

\section{CONCLUSION}

The current evidence base available for different treatment options provides ambiguous results mainly because of the study designs and the end points assessed. This is also reflected in the different national treatment guidelines that were based on relatively weak evidence. The systematic review highlighted the lacuna and methodologic deficiency in early clinical evidence and an update on different therapeutic management guidelines. The results of the ongoing clinical studies and well-designed realworld studies will improve the evidence base and may lead to further evolution of treatment guidelines with the addition of more therapeutic options. Furthermore, a consensus on the 
appropriate end points for efficacy in different categories of patient may also improve the quality of evidence in case of future pandemics of respiratory viruses.

\section{ACKNOWLEDGEMENTS}

Funding. No funding or sponsorship was received for this study or publication of this article. The Rapid Service Fee was funded by the Indegene Pvt Ltd.

Authorship. All named authors meet the International Committee of Medical Journal Editors (ICMJE) criteria for authorship for this article, take responsibility for the integrity of the work as a whole, and have given their approval for this version to be published.

Disclosures. Kaushik Subramanian, Anuradha Nalli, Vinitha Senthil, Saurabh Jain, Aravind Nayak and Amit Bhat are employees of Indegene Pvt Ltd.

Compliance with Ethics Guidelines. This article is based on previously conducted studies and does not contain any studies with human participants or animals performed by any of the authors.

\section{REFERENCES}

1. Coronavirus Disease (COVID-19)—events as they happen [Internet]. Available from: https://www. who.int/emergencies/diseases/novel-coronavirus2019/events-as-they-happen. Cited 14 May 2020.

2. WHO. Coronavirus disease (COVID-19) Situation Report- 114 [Internet]. Available from: https:// www.who.int/docs/default-source/coronaviruse/ situation-reports/20200513-covid-19-sitrep-114. pdf?sfvrsn=17ebbbe_4. May 2020.

3. Ye Z-W, Jin D-Y. Diagnosis, treatment, control and prevention of SARS-CoV-2 and coronavirus disease 2019: back to the future. Sheng Wu Gong Cheng Xue Bao. 2020;36:571-92.
4. Li X, Geng M, Peng Y, Meng L, Lu S. Molecular immune pathogenesis and diagnosis of COVID-19. J Pharm Anal. 2020;10:102-8.

5. Guan W, Ni Z, Hu Y, Liang W, Ou C, He J, et al. Clinical characteristics of coronavirus disease 2019 in China. N Engl J Med. 2020;382:1708-20.

6. Li H, Liu L, Zhang D, Xu J, Dai H, Tang N, et al. SARS-CoV-2 and viral sepsis: observations and hypotheses. The Lancet. 2020;395:1517-20.

7. Liu J, Li S, Liu J, Liang B, Wang X, Wang H, et al. Longitudinal characteristics of lymphocyte responses and cytokine profiles in the peripheral blood of SARS-CoV-2 infected patients. medRxiv. 2020;2020. 02.16.20023671.

8. Middeldorp S, Coppens $M$, Haaps TF van, Foppen M, Vlaar AP, Müller MCA, et al. Incidence of venous thromboembolism in hospitalized patients with COVID-19. Journal of Thrombosis and Haemostasis [Internet]. Available from: https://onlinelibrary. wiley.com/doi/abs/10.1111/jth.14888. Cited 24 Jul 2020.

9. Nikolich-Zugich J, Knox KS, Rios CT, Natt B, Bhattacharya D, Fain MJ. SARS-CoV-2 and COVID-19 in older adults: what we may expect regarding pathogenesis, immune responses, and outcomes. GeroScience. 2020;1-10.

10. Team TNCPERE. The Epidemiological characteristics of an outbreak of 2019 novel Coronavirus diseases (COVID-19)—China, 2020. CCDCW. 2019;2020(2):113-22.

11. Gates B. Responding to Covid-19-a once-in-acentury pandemic? N Engl J Med. 2020;382:1677-9.

12. Guyatt G, Oxman AD, Akl EA, Kunz R, Vist G, Brozek J, et al. GRADE guidelines: 1. IntroductionGRADE evidence profiles and summary of findings tables. J Clin Epidemiol. 2011;64:383-94.

13. Rosa SGV, Santos WC. Clinical trials on drug repositioning for COVID-19 treatment. Rev Panam Salud Publica [Internet]. 2020;44. Available from: https://www.ncbi.nlm.nih.gov/pmc/articles/ PMC7105280/. Cited 17 May 2020.

14. Vincent MJ, Bergeron E, Benjannet S, Erickson BR, Rollin PE, Ksiazek TG, et al. Chloroquine is a potent inhibitor of SARS coronavirus infection and spread. Virol J. 2005;2:69.

15. Wang M, Cao R, Zhang L, Yang X, Liu J, Xu M, et al. Remdesivir and chloroquine effectively inhibit the recently emerged novel coronavirus (2019-nCoV) in vitro. Cell Res. 2020;30:269-71. 
16. Yao X, Ye F, Zhang M, Cui C, Huang B, Niu P, et al. In vitro antiviral activity and projection of optimized dosing design of hydroxychloroquine for the treatment of severe acute respiratory syndrome Coronavirus 2 (SARS-CoV-2). Clin Infect Dis [Internet]. Available from: https://academic.oup.com/ cid/advance-article/doi/10.1093/cid/ciaa237/ 5801998. Cited 17 May 2020

17. Gautret P, Lagier J-C, Parola P, Hoang VT, Meddeb $\mathrm{L}$, Mailhe $\mathrm{M}$, et al. Hydroxychloroquine and azithromycin as a treatment of COVID-19: results of an open-label non-randomized clinical trial. Int J Antimicrob Agents. 2020;105949.

18. Chen Z, Hu J, Zhang Z, Jiang S, Han S, Yan D, et al. Efficacy of hydroxychloroquine in patients with COVID-19: results of a randomized clinical trial. medRxiv. 2020;2020.03.22.20040758.

19. Million M, Lagier J-C, Gautret P, Colson P, Fournier P-E, Amrane S, et al. Early treatment of COVID-19 patients with hydroxychloroquine and azithromycin: a retrospective analysis of 1061 cases in Marseille, France. Travel Med Infect Dis 2020;101738.

20. Chorin E, Dai M, Shulman E, Wadhwani L, Cohen $\mathrm{RB}$, Barbhaiya C, et al. The QT interval in patients with SARS-CoV-2 infection treated with Hydroxychloroquine/Azithromycin. medRxiv. 2020;2020. 04.02.20047050.

21. Chen J, Liu D, Liu L, Liu P, Xu Q, Xia L, et al. A pilot study of hydroxychloroquine in treatment 1129 of patients with common coronavirus disease-19 (COVID-19) [Internet]. Epistemonikos. Available from: https://www.epistemonikos.org/en/docu ments/30d0f2552ff5342fbfcd1b09e9bac66d1325b4 67

22. Molina JM, Delaugerre C, Le Goff J, Mela-Lima B, Ponscarme D, Goldwirt L, et al. No evidence of rapid antiviral clearance or clinical benefit with the combination of hydroxychloroquine and azithromycin in patients with severe COVID-19 infection. Med Mal Infect [Internet]. 2020; Available from: https://www.ncbi.nlm.nih.gov/pmc/ articles/PMC7195369/. Cited 17 May 2020

23. Magagnoli J, Narendran S, Pereira F, Cummings T, Hardin JW, Sutton SS, et al. Outcomes of hydroxychloroquine usage in United States veterans hospitalized with Covid-19. medRxiv. 2020;2020.04.16. 20065920.

24. Tang W, Cao Z, Han M, Wang Z, Chen J, Sun W, et al. Hydroxychloroquine in patients mainly with mild to moderate COVID-19: an open-label, randomized, controlled trial. medRxiv. 2020;2020.04. 10.20060558 .
25. Cao Y, Deng Q, Dai S. Remdesivir for severe acute respiratory syndrome coronavirus 2 causing COVID-19: An evaluation of the evidence. Travel Med Infect Dis [Internet]. 2020; Available from: https://www.ncbi.nlm.nih.gov/pmc/articles/ PMC7151266/. Cited 17 May 2020.

26. de Wit E, Feldmann F, Cronin J, Jordan R, Okumura A, Thomas T, et al. Prophylactic and therapeutic remdesivir (GS-5734) treatment in the rhesus macaque model of MERS-CoV infection. PNAS. 2020;117:6771-6.

27. Sheahan TP, Sims AC, Leist SR, Schäfer A, Won J, Brown AJ, et al. Comparative therapeutic efficacy of remdesivir and combination lopinavir, ritonavir, and interferon beta against MERS-CoV. Nat Commun. 2020;11:222.

28. Holshue ML, DeBolt C, Lindquist S, Lofy $\mathrm{KH}$, Wiesman J, Bruce H, et al. First case of 2019 novel coronavirus in the United States. N Engl J Med. 2020;382:929-36.

29. Grein J, Ohmagari N, Shin D, Diaz G, Asperges E, Castagna A, et al. Compassionate Use of Remdesivir for Patients with Severe Covid-19. N Engl J Med. 2020.

30. Wang Y, Zhang D, Du G, Du R, Zhao J, Jin Y, et al. Remdesivir in adults with severe COVID-19: a randomised, double-blind, placebo-controlled, multicentre trial. The Lancet. 2020;395:1569-78.

31. Halpin DMG, Faner R, Sibila O, Badia JR, Agusti A. Do chronic respiratory diseases or their treatment affect the risk of SARS-CoV-2 infection? Lancet Respir Med. 2020;8:436-8.

32. Matsuyama S, Kawase M, Nao N, Shirato K, Ujike M, Kamitani W, et al. The inhaled corticosteroid ciclesonide blocks coronavirus RNA replication by targeting viral NSP15. bioRxiv. 2020;2020.03.11. 987016.

33. Russell B, Moss C, Rigg A, Van Hemelrijck M. COVID-19 and treatment with NSAIDs and corticosteroids: should we be limiting their use in the clinical setting? Ecancermedicalscience [Internet]. 2020;14. Available from: https://www.ncbi.nlm. nih.gov/pmc/articles/PMC7105332/. Cited 17 May 2020.

34. Zha L, Li S, Pan L, Tefsen B, Li Y, French N, et al. Corticosteroid treatment of patients with coronavirus disease 2019 (COVID-19). Med J Aust. 2020;212:416-20.

35. Yang Z, Liu J, Zhou Y, Zhao X, Zhao Q, Liu J. The effect of corticosteroid treatment on patients with coronavirus infection: a systematic review and meta-analysis. J Infect [Internet]. 2020; Available 
from: https://www.ncbi.nlm.nih.gov/pmc/articles/ PMC7195158/. Cited 17 May 2020.

36. Wang Y, Jiang W, He Q, Wang C, Wang B, Zhou P, et al. Early, low-dose and short-term application of corticosteroid treatment in patients with severe COVID-19 pneumonia: single-center experience from Wuhan, China. medRxiv. 2020;2020.03.06. 20032342.

37. Lu X, Chen T, Wang Y, Wang J, Zhang B, Li Y, et al. Adjuvant corticosteroid therapy for critically ill patients with COVID-19. medRxiv. 2020;2020.04. 07.20056390 .

38. Shen C, Wang Z, Zhao F, Yang Y, Li J, Yuan J, et al. Treatment of 5 critically ill patients with COVID-19 with convalescent plasma. JAMA. 2020;323:1582-9.

39. Duan K, Liu B, Li C, Zhang H, Yu T, Qu J, et al. Effectiveness of convalescent plasma therapy in severe COVID-19 patients. Proc Natl Acad Sci USA. 2020;117:9490-6.

40. Ahn JY, Sohn Y, Lee SH, Cho Y, Hyun JH, Baek YJ, et al. Use of Convalescent Plasma Therapy in Two COVID-19 Patients with Acute Respiratory Distress Syndrome in Korea. J Korean Med Sci [Internet]. 2020;35. Available from: https://www.ncbi.nlm. nih.gov/pmc/articles/PMC7152526/. Cited 18 May 2020 .

41. Xu X, Han M, Li T, Sun W, Wang D, Fu B, et al. Effective treatment of severe COVID-19 patients with tocilizumab. PNAS. 2020;117:10970-5.

42. Luna GD, Habibi A, Deux J-F, Colard M, d'Orengiani A-LPH d'Alexandry, Schlemmer F, et al. Rapid and severe Covid-19 pneumonia with severe acute chest syndrome in a sickle cell patient successfully treated with tocilizumab. American Journal of Hematology [Internet]. Available from: https:// onlinelibrary.wiley.com/doi/abs/10.1002/ajh. 25833. Cited 21 May 2020.

43. Ye X-T, Luo Y-L, Xia S-C, Sun Q-F, Ding J-G, Zhou Y, et al. Clinical efficacy of lopinavir/ritonavir in the treatment of Coronavirus disease 2019. Eur Rev Med Pharmacol Sci. 2020;24:3390-6.

44. Zhu Z, Lu Z, Xu T, Chen C, Yang G, Zha T, et al. Arbidol monotherapy is superior to lopinavir/ritonavir in treating COVID-19. J Infect [Internet]. 2020; Available from: https://www.ncbi.nlm.nih. gov/pmc/articles/PMC7195393/. Cited 18 May 2020

45. Li Y, Xie Z, Lin W, Cai W, Wen C, Guan Y, et al. An exploratory randomized controlled study on the efficacy and safety of lopinavir/ritonavir or arbidol treating adult patients hospitalized with mild/moderate COVID-19 (ELACOI). medRxiv. 2020;2020.03.19.20038984.

46. Institute of Medicine (US) Committee on Standards for Developing Trustworthy Clinical Practice Guidelines. Clinical Practice Guidelines We Can Trust [Internet]. Graham R, Mancher M, Miller Wolman D, Greenfield S, Steinberg E, editors. Washington (DC): National Academies Press (US); 2011;Available from: https://www.ncbi.nlm.nih. gov/books/NBK209539/. Cited 24 Jul 2020.

47. Clinical management of severe acute respiratory infection (SARI) when COVID-19 disease is suspected [Internet]. WHO; 2020. Available from: https://apps.who.int/iris/handle/10665/331446. Cited 24 Jul 2020.

48. Clinical management of COVID-19, interim guidance [Internet]. 2020. Available from: https://www. who.int/publications/i/item/clinical-managementof-covid-19

49. Commissioner O of the. Emergency Use Authorization. FDA [Internet]. 2020 [; Available from: https://www.fda.gov/emergency-preparedness-andresponse/mcm-legal-regulatory-and-policy-framework/emergency-use-authorization. Cited $24 \mathrm{Jul}$ 2020 .

50. Commissioner O of the. Coronavirus (COVID-19) Update: FDA Issues Emergency Use Authorization for Potential COVID-19 Treatment [Internet]. FDA. 2020. Available from: https://www.fda.gov/newsevents/press-announcements/coronavirus-covid19-update-fda-issues-emergency-use-authorizationpotential-covid-19-treatment. Cited 24 Jul 2020.

51. COVID-19 Treatment Guidelines Panel. Coronavirus Disease 2019 (COVID-19) Treatment Guidelines. [Internet]. National Institutes of Health. Available from: https://www. covid19treatmentguidelines.nih.gov/. Cited Jul 2020

52. Infectious Diseases Society of America Guidelines on the Treatment and Management of Patients with COVID-19 [Internet]. Available from: https://www. idsociety.org/practice-guideline/covid-19guideline-treatment-and-management/. Cited Apr 2020 .

53. Jin Y-H, Cai L, Cheng Z-S, Cheng H, Deng T, Fan $\mathrm{Y}-\mathrm{P}$, et al. A rapid advice guideline for the diagnosis and treatment of 2019 novel coronavirus (2019$\mathrm{nCoV}$ ) infected pneumonia (standard version). Mil Med Res. 2020;7:4.

54. Revised Guidelines on Clinical Management of COVID-19 [Internet]. Available from: https:// www.mohfw.gov.in/pdf/RevisedNationalClinical 
ManagementGuidelineforCOVID1931032020.pdf. Cited Mar 2020.

55. Directorate General of Health Services. Revised advisory on the use of Hydroxychloroquine (HCQ) as prophylaxis for COVID-19 infection [Internet]. Available from: https://www.mohfw.gov.in/pdf/ Revisedadvisoryontheuseofhydro xychloroquineasprophylaxisfor SARSCOVID19infection.pdf. Cited May 2020.

56. Directorate General of Health Services. Revised Guidelines on Clinical Management of COVID-19 [Internet]. Available from: https://www.mohfw.gov.in/. Cited Mar 2020.

57. Dagens A, Sigfrid L, Cai E, Lipworth S, Cheng V, Harris E, et al. Scope, quality, and inclusivity of clinical guidelines produced early in the covid-19 pandemic: rapid review. BMJ [Internet]. 2020;369. Available from: https://www.bmj.com/content/ 369/bmj.m1936. Cited 24 Jul 2020.

58. RECOVERY Collaborative Group, Horby P, Lim WS, Emberson JR, Mafham M, Bell JL, et al. Dexamethasone in hospitalized patients with Covid-19preliminary report. N Engl J Med 2020.
59. Beigel JH, Tomashek KM, Dodd LE, Mehta AK, Zingman BS, Kalil AC, et al. Remdesivir for the treatment of Covid-19-preliminary report. N Engl J Med 2020.

60. Desai A, Gyawali B. Endpoints used in phase III randomized controlled trials of treatment options for COVID-19. EClinicalMedicine. 2020;23:100403.

61. Mehra MR, Desai SS, Ruschitzka F, Patel AN. RETRACTED: Hydroxychloroquine or chloroquine with or without a macrolide for treatment of COVID-19: a multinational registry analysis. Lancet. 2020 .

62. Oransky AI. French hydroxychloroquine-COVID-19 study withdrawn [Internet]. Retraction Watch. 2020. Available from: https://retractionwatch.com/ 2020/05/21/french-hydroxychloroquine-covid-19study-withdrawn. Cited 30 Jun 2020.

63. COVID-19 vaccine tracker [Internet]. Available from: https://www.raps.org/news-and-articles/ news-articles/2020/3/covid-19-vaccine-tracker.

Cited 25 Jul 2020. 